\title{
High Voltage Transmission Line Environment Characteristics of Image Edge
}

\author{
Weihua Niu \& Jiangman Zhao \\ School of North China Electric Power University, Baoding 071000, China \\ Peng Zhao \\ State Grid Hebei electric power company, China
}

\begin{abstract}
According to different environment around the high voltage transmission lines, the analysis of image characteristics under different environment, different environment image segmentation method applied to image feature extraction method and edge of image, and compare the effect of two kinds of methods, reach to the effect of rapid extraction and identification of obstacles.
\end{abstract}

KEYWORD: High voltage transmission line; edge detection; Image characteristic

\section{INTRODUCTION}

Transmission lines based on line installation location is usually divided into cable transmission lines and overhead transmission lines (Chi JN et al, 2004). Cable transmission lines generally buried underground cable tunnel, it is extremely rare to see. The general frame on the tower overhead transmission line (Freeman H, 1970), and appeared in the sky, it is easy to see, so easily damaged overhead transmission lines (Donoho DL et al, 2000). According to the power facility protection ordinance provides high voltage transmission lines and obstacles keep a safe distance. In recent years, with China's rapid economic development, urban and rural residents' living standard unceasing enhancement, the building construction increasing (Hough PVC, 1962), large-scale construction vehicle, and trees too fast growth poses a serious threat to high voltage transmission lines, the barrier of high voltage line and there is no doubt that became a threat to the power grid safe operation, endangering public security, hurt the people life "unabomber" and "safe killer" (Leica GeoSystems, 2007), it will threaten high voltage transmission line environment is divided into three categories, respectively, for the community construction, environmental greening (Hu Shen et al, 2010), largescale construction machinery causing serious harm of high voltage transmission line. In order to solve this problem, puts forward the monocular vision technology, extract image information by the camera to the scene environment, through the analysis of image information research, and realize the early warning effect.

\section{IMAGE EDGE DETECTION TRANSMISSION LINES BASED ON LOG RESEARCH}

High voltage transmission lines under the structure of the building, has a great threat for high voltage transmission lines, and from the high tension line not within a safe distance from buildings, form great threat to the safety of the people. Through an analysis on the transmission line and the image characteristics of buildings, selecting suitable image feature extraction method, floor of building, as a general rule, be under construction, the color is deep than the sky background, therefore USES the LoG operator edge detection algorithm, the result of edge extraction based on LoG operator is superior to Roberts operator and Sobel operator, especially edge is more complete, accurate location, there is a better ability to resist noise.

In order to reduce the influence of noise on the edge, the first image should be smooth, low pass filtering LoG method use the gaussian function as a low pass filter. Gaussian function as follows:

$$
\dot{G}(x, y)=e^{-\frac{x^{2}+y^{2}}{2 \delta^{2}}}
$$

Type in the delta determines the smooth degree of the images. Using the gaussian function of image filtering and image filtering result of second order differential operation process, can convert first to second order differential of gaussian function, using gaussian function of second order differential results for convolution operation, the process is expressed by the following mathematical formula:

$$
\nabla^{2}[I(x, y) \otimes G(x, y)]=\nabla^{2} G(x, y) \otimes I(x, y)
$$

LoG operator is pass filter. In practice, can be 
$\nabla^{2} \mathbf{G}\left(\mathrm{x}_{\mathrm{r}} \mathrm{y}\right)$ is simplified to:

$\nabla^{2} G(x, y)=\mathrm{K}\left(2-\frac{x^{2}+y^{2}}{\delta^{2}}\right) \cdot e^{-\frac{x^{2}+y^{2}}{2 \delta^{2}}}$

In parameter design, the delta value is bigger, tends to smooth the image; the delta values is small, tend to be sharpening the image. Usually should be according to the characteristics of the image and choose the right through the experiment of the delta. Here through the delta best-fit values, used in the extraction of image characteristics under the environment of high voltage transmission line building, through the experiment to find the best value of 1.6 to 1.6 is the best. The selection of $\mathrm{K}$ should make each array element is positive and make the sum of all arrays are zero. Use of the LoG operator for edge detection steps are as follows:

(1) Using Laplacian of gaussian filter of image filtering, filtering the image.

(2) Zero detection, have been carried out to get the image of the specific method is: assume your image first order differential of each pixel of the image as the $P[i, j], L[I, j]$ for its Laplace values, the meaning of the $\mathrm{P}$ and $\mathrm{L}$ as shown.

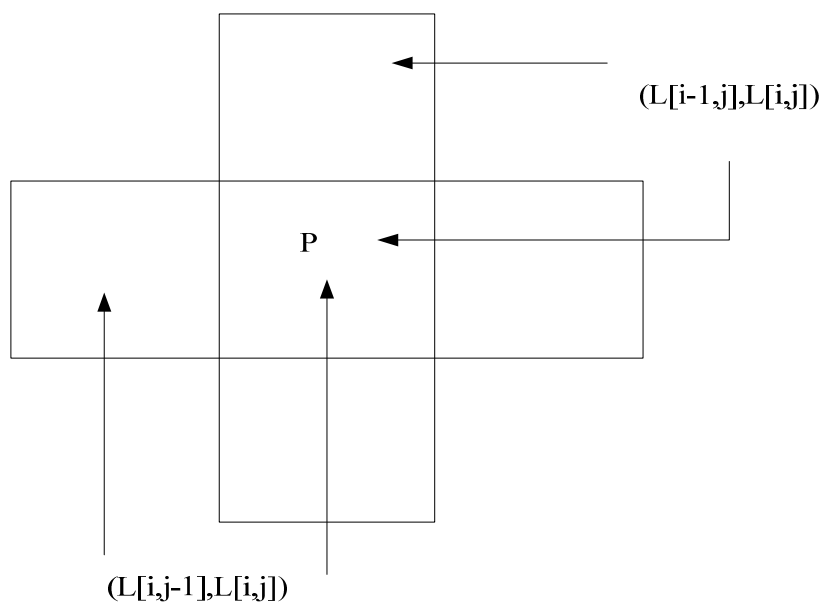

(3) The judge in accordance with the following rules:

If $\mathrm{L}[\mathrm{I}, \mathrm{j}]=0$, then the number of $(\mathrm{L}[\mathrm{i}-1, \mathrm{j}], \mathrm{L}[\mathrm{i}+1$, $j])$ or $(L[I, j-1], L[I, j+1])$ is involved in the plus or minus the opposite two Numbers. As long as the one of the two Numbers contain plus or minus of two Numbers to the contrary, the $\mathrm{P} / \mathrm{I}, \mathrm{j}$ corresponds to the first-order differential score is above a certain threshold, if it is, the P/I and $\mathrm{j}$ is the edge points, otherwise not. If $L[I, j)$ is not zero, then the four number of ([i, j], L[i-1,j], (L[i,j], L[i+1,j]), (L[i, j], $\mathrm{L}[\mathrm{i}, \mathrm{j}-1]),(\mathrm{L}[\mathrm{i}, \mathrm{j}], \mathrm{L}[\mathrm{i}, \mathrm{j}+1])$ whether there is included in plus or minus the opposite value. If so, with zero crossing near the $P[i, j]$. And then see the $P[i, j]$ corresponds to the first-order differential score is above a certain threshold, if it is, will be th $P[i, j] j$ as edge points.

\section{THE EXPERIMENTAL RESULTS}

\subsection{The Log operator to detect}

For high voltage transmission line under different environment image experiment result is shown in figure 1-1, figure 1-2, and figure. 1-3:

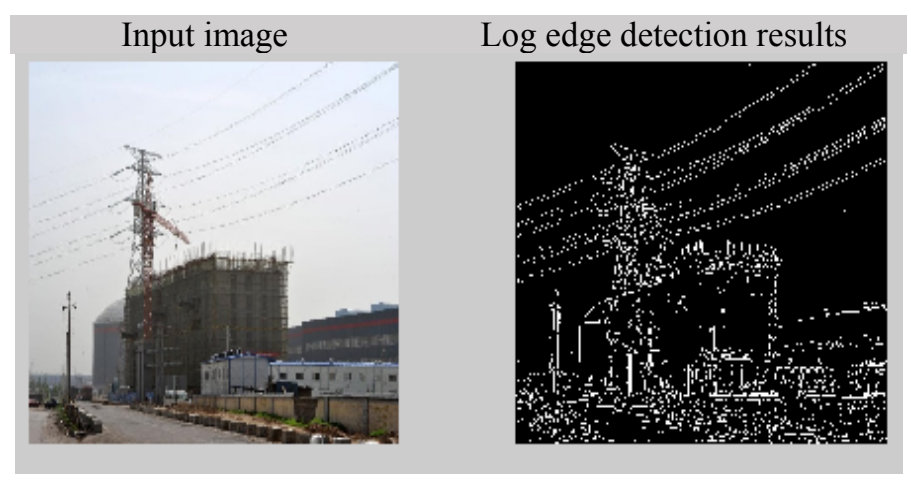

Figure. 1-1 Log operator transmission lines image measuring building environment

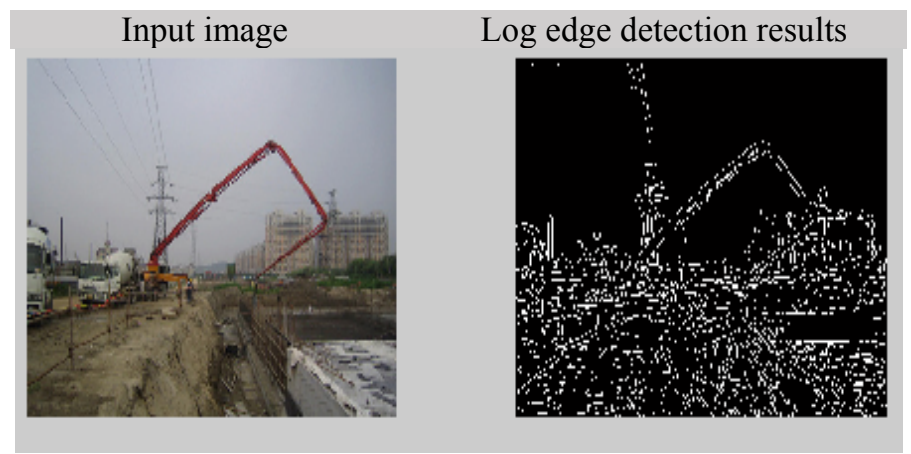

Figure 1-2 Log operator to detect large vehicles under high voltage transmission line image

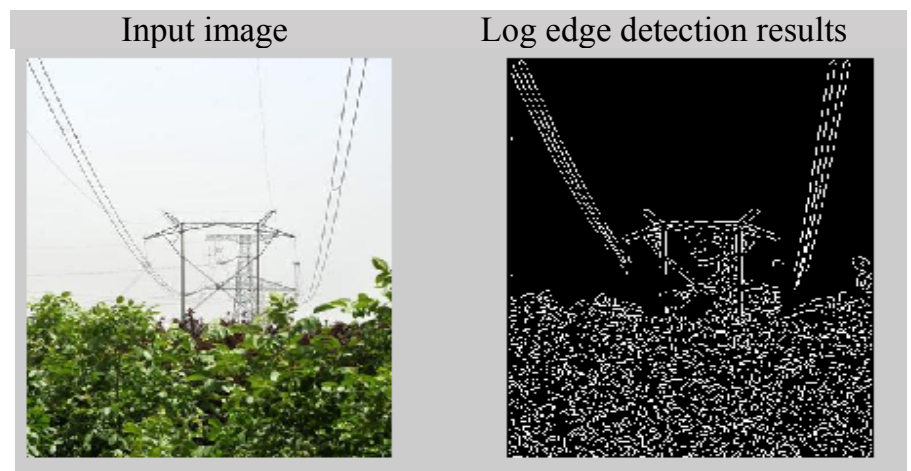

Figure 1-3 test Log operator trees environment image transmission lines

Through the experiment, under the environment of high voltage transmission line based on Log operator edge detection image characteristics, choose the best suitable value delta, experimental analysis to find the best value of 1.8. See the image to detect the edge is more, are greatly influenced by uneven illumination and background gray. 


\subsection{Threshold segmentation method}

Through the analysis of the transmission line environment image characteristics, the study found that the image of the sky color as the background color, the rest for prospects or target, the target with a specific outline and color, in order to identify and analyze the image of the target, need to be separated out from the image, on the basis of this measure to use and the target image. Image segmentation into each area of the image to extract the interested target.

The definition of image segmentation to set $\mathrm{R}$ represents the entire image region, into $4 \mathrm{R}$ to $\mathrm{R}$ segmentation can be seen as $\mathrm{N}$ the loophole that meet the following conditions set R1, R2,.. RN:

(1) $\bigcup_{\tau=4}^{Z} R_{4}=R$;

(2) for all the $I, j$, when $I$ indicates $j$, meet $R_{-} i$ studying $\mathrm{R} \mathrm{j}=$;

(3) for $\mathrm{I}=1,2, \ldots, \mathrm{N}, \mathrm{P}\left(\mathrm{R} \_\mathrm{i}\right)=\mathrm{TRUE}$;

(4) for I indicates $j, P\left(R_{-} i\right.$ studying $\left.R_{-} j\right)=$ FALSE;

(5) for $I=1,2, \ldots, N, R \_i$ is along with the area.

On the type of $\mathrm{P}$ for elements in the collection of all R_i logical predicate, represents an empty set. By definition: a pair of image segmentation results, all sub area and sets should be able to include all of the image pixels, and the segmentation results, each area is non-overlapping, namely each pixel can't belong to different areas at the same time. Belong to the same area of the pixel should meet some of the same attributes, and different areas have different characteristics. Segmentation results in the same region pixels should be connected.

The analysis of features of image, target and the background color the sky with different grayscale range, choose to use a threshold segmentation method, threshold segmentation method is divided into two kinds of global threshold and partial threshold. If every pixel in the process of segmentation of image using the same threshold, it is called a global threshold value method; If the whole image not all pixels using threshold value are the same, it is called a local threshold. The selection of threshold is the key to threshold segmentation method. Consider the following situation: images have a shadow, uneven illumination, contrast different everywhere, there are sudden noise and background gray level change, if only a fixed global threshold value for the whole image segmentation, is due to the situation that is not balance in all parts of the image segmentation result is affected. There is a solution is to use a set of threshold pixel position related to all parts of the image segmentation, respectively. The coordinate related threshold is called adaptive threshold.

For high voltage transmission line under different environment image experiment result is shown in figure 1-4, figure 1-5, as shown in figure 1-6:

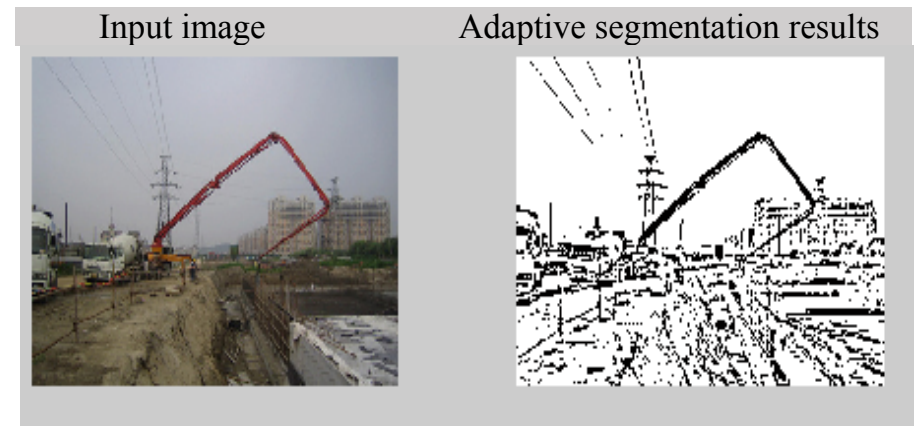

Figure 1-4 threshold segmentation under the environment of building high-voltage transmission lines image

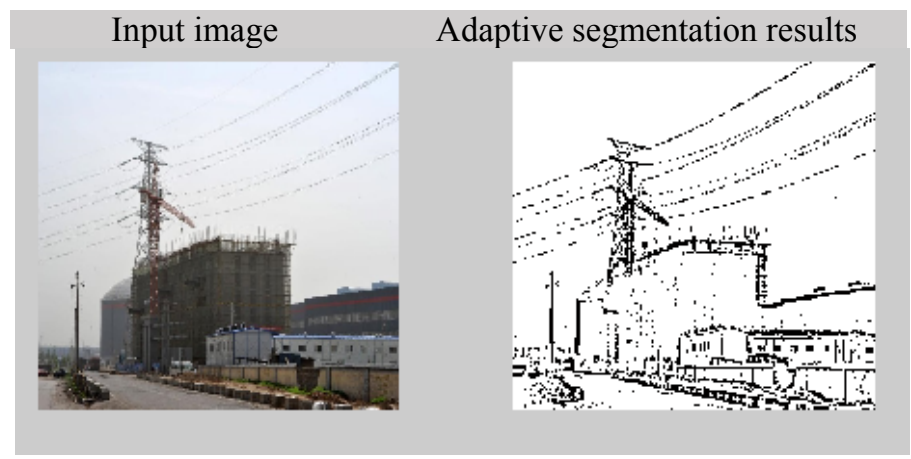

Figure 1-5 threshold segmentation large vehicle transmission lines under the image

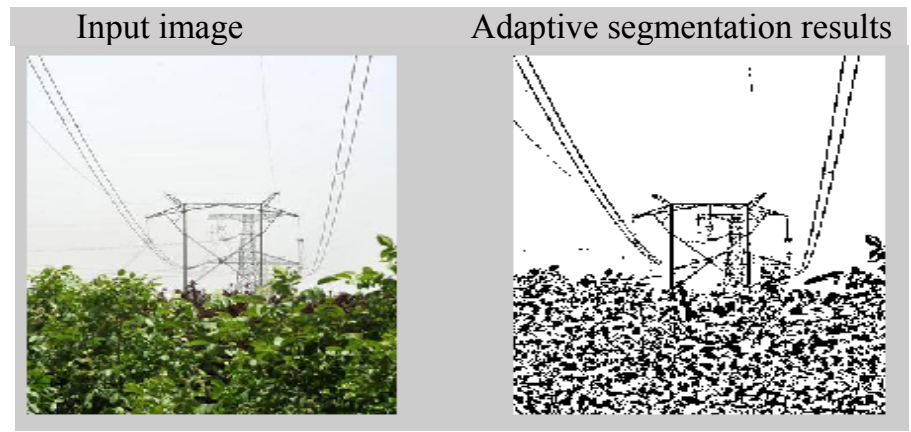

Figure 1-6 threshold segmentation trees environment image transmission lines

Through the experiment, the function adaptive threshold (IM, ws, C, tm) function for the adaptive image segmentation, IM for segmentation of the original image, the ws for average filtering window size, $\mathrm{C}$ according to the constant for application in transmission line environment experiment to select parameters of 0.048 , tm switch variables, $\mathrm{tm}=1$, median filtering to average filtering $\mathrm{tm}=0$. Application of transmission line under different environment image local threshold segmentation, the background and the target can better segmentation.

\section{SUMMARY}

Through the experiment, under the environment of high voltage transmission line based on Log operator edge detection image characteristics, choose the 
best suitable value delta, experimental analysis to find the best value of 1.8. See the image to detect the edge is more, are greatly influenced by uneven illumination and background gray.

\section{REFERENCES}

Chi JN, Xu XH. Research on simultaneous localization and mapping of mobile robot. Robot, 2004, 26(1): 92-96.

Donoho DL, Huo XM. Beamlets and multiscale image analysis. Springer Lecture Notes in Computer Science and Enginering: Multiresolution Methods, 2000.

Freeman H. Boundary Encoding and Processing. BS Lipkin, A Rosenfeld. Picture Processingand Psychopictorics, Academic, New York, 1970, 12(6): 241-266.

Hough PVC. A Method and Means For Recognizing Complex Patterns. USA, 069654. 1962, 23(6): 7-21.

HU SHEN, PAN ZHEN KUAN. Research on Edge Detection Algorithm Based on Shorten Wavelet Packet[J]. College of Information Technology Qingdao University, 2010, 23(2): 23-27.

Leica GeoSystems. ADS40 Technical Reference Manual. Switzerland: Leica Geosyetems, 2007. 